\title{
A comparative study of diagnostic tests and conjunctival impression cytology in patients with dry eye disease and healthy controls
}

\author{
Rajesh Sharma ${ }^{1 *}$, Rajeev Tuli², R K Sharma ${ }^{3}$, Rashmi Kaul Raina ${ }^{4}$ \\ $\left\{{ }^{1}\right.$ Resident, ${ }^{3}$ Professor, ${ }^{2}$ Professor \& HOD, Department of Ophthalmology $\{$ \{4Associate Professor \& HOD, Department of Pathology $\}$ \\ Dr. Rajendra Prasad Government Medical College, Kangra at Tanda, Himachal Pradesh, INDIA. \\ Email: suhi1551@gmail.com
}

Abstract Background: Dry eye disease is a common problem worldwide and can reduce the working efficiency of an individual. Dry eye disease (DED) is a distressing ocular condition. Due to its multifactorial nature, clinical and biological signs of DED can be inconsistent and sometimes discordant with symptomatology. Various tests are available for diagnosing DED. Aim and objective: To evaluate dry eye diagnostic tests and conjunctival impression cytology in patients with dry eye disease and healthy controls. Methodology: This was a case-control, non-interventional study to evaluate dry eye diagnostic tests and conjunctival impression cytology in patients with dry eye disease and healthy controls. A total of 142 subjects (74 cases and 68 controls), based on the inclusion and exclusion criteria, from 20 years of age to 60 years were included in this study. Patients were subjected to detailed history and examination using standardized protocol and performa. Schirmer, TBUT, BME and CIC were carried out in cases and controls. Data was analysed with appropriate statistical tests. Results and discussion: TBUT and BME were better predictors of morphological and cytological changes in the conjunctiva than Schirmer. Moreover, TBUT was more sensitive and specific than BME and Schirmer for diagnosis of dry eye. The accuracy of tear function tests in predicting morphological and cytological changes in the conjunctiva was TBUT $>$ BME $>$ Schirmer. The diagnostic accuracy was $\operatorname{TBUT}(0.775)>\operatorname{OSDI}(\mathrm{AUC}=0.730)>\mathrm{BME}(\mathrm{AUC}=0.693)>\mathrm{Schirmer}$ $(\mathrm{AUC}=0.853)$.

Key Word: conjunctival impression cytology.

*Address for Correspondence:

Dr Rajesh Sharma, Palampur, Resident, Department of Ophthalmology, Dr. Rajendra Prasad Government Medical College, Kangra At Tanda, Himachal Pradesh Pin 176001. INDIA.

Email: suhi1551@gmail.com

Received Date: 02/05/2020 Revised Date: 19/05/2020 Accepted Date: 23/06/2020

DOI: https://doi.org/10.26611/10091512

This work is licensed under a Creative Commons Attribution-NonCommercial 4.0 International License. $(\mathbf{c c})$ EY-NC

\begin{tabular}{|l|l|}
\hline \multicolumn{2}{|c|}{ Access this article online } \\
\hline Quick Response Code: & Website: \\
\cline { 1 - 2 } & www.medpulse.in \\
& \\
\hline
\end{tabular}

\section{INTRODUCTION}

Dry eye disease is one of the most frequently encountered ocular morbidities and is one of the most common complaints seen by ophthalmic specialists ${ }^{1}$, making it a growing public health problem. It is a condition that causes considerable morbidity and reduces the quality of the life of an individual. It affects $5-35 \%$ of the global adult population ${ }^{2}$. According to the International Dry Eye Workshop (DEWS2007), dry eye disease is a multifactorial disease of tears and the ocular surface. It results in symptoms of discomfort, visual disturbances and tear-film instability with potential damage to the ocular surface. It is accompanied by increased osmolarity of the tear film and inflammation of the ocular surface ${ }^{3}$. Diagnosis of dry eye syndrome is essentially clinical. It is based on the presence of typical symptoms - mostly common with other ophthalmic diseases, clinical examination and some specific tests, which evaluate the severity of the disease, guide the follow-up and can help with the therapeutic decisions. A combination of various 
subjective and objective measurements are often used to determine the presence and/or severity of dry eye in an individual. ${ }^{4,5}$ In addition, it has been recognised, particularly in moderate/mild dry eye, that diagnostic tests are prone to disagree and give conflicting results. Begley et al. ${ }^{5}$ previously reported that ocular surface staining did not always correlate with patient's dry eye symptoms, while Kallarackal et al. ${ }^{6}$ found a poor correlation between Schirmer's test and tear break up time in dry eye patients. At present there is no general consensus for the criterion for the diagnosis of dry eye. In the absence of an agreed 'gold standard', no set of criteria has been universally adopted which can result in unpredictable variations in test results and diagnosis between studies, as per the International Dry Eye Workshop, 2007.7,8 Staining with vital dyes like Rose Bengal measures the extent of damage to the ocular surface, but this method may not detect mild cases. Conjunctival excision biopsy can be used for the histo-pathological diagnosis of dry eye. However, this procedure is invasive and therefore may not be accepted by patients. In clinical practice, not all centers may be equipped to perform the biopsy ${ }^{9}$. Impression cytology is a useful non-invasive technique, in which the first or the two outermost layers of the ocular surface epithelium are removed while preserving cellular morphology and studied to determine the state of the conjunctival surface and to classify the severity of squamous metaplasia. This technique allows the investigator to assess epithelial cell morphology, examine cytoplasmic and nuclear characteristics and quantify the goblet cell population in the conjunctiva. Impression cytology is more sensitive than clinical and laboratory tests, and has the advantage of being able to detect squamous metaplasia before keratinization is clinically detectable. ${ }^{10,11}$ Conjunctival impression cytology (CIC) has been widely used as a method for evaluating the ocular surface and superficial cells layers in the diagnosis and follow-up after treatment of several ocular surface tumors of both epithelial and melanocytic origin. ${ }^{12}$ Applications of impression cytology include the etiological diagnosis of various ocular surface disorders, documenting sequential changes in the conjunctival and corneal surface over time, monitoring effects of treatment and staging conjunctival squamous metaplasia, and as an investigational tool for analysing ocular surface disease with immunostaining and DNA analysis. ${ }^{13}$ It is minimally invasive, relatively easy to perform and yields reliable information about the area sampled with minimal discomfort to the patient. ${ }^{14-16}$ But it is still not used as a first line investigation in dry eye disease. We intended to study conjunctival impression cytology in view of overall paucity of data.

\section{MATERIAL AND METHODS}

A total of 142 patients (74 cases and 68 controls) successfully completed the study. Informed consent was obtained from all patients prior to enrolment in this study. For cases, patients from 20 years up to the age of 60 years, either sex who fulfilled the inclusion/exclusion criteria, were included. For controls, patients from 20 years up to the age of 60 years who fulfilled the inclusion/exclusion criteria were included. Study area was the Department of Ophthalmology and the Department of Pathology of Dr.Rajendra Prasad Government Medical College, Kangra at Tanda. All the subjects who were included as cases or controls were asked to respond to OSDI questionnaire. Based on their OSDI scores, patients were categorized as having a normal ocular surface( $0-12$ points) or as having mild(13-22 points), moderate(23-32 points), and severe(33-100 points) ocular surface disease. The participants attaining the score $0-12$ were selected as controls while those with score 13-100 were taken as dry eye patients. The enrolled participants were subjected to detailed medical history and clinical examination as per performa. Exclusion criteria: 1.Patients with past or present ocular diseases such as current infections including herpetic eye disease, corneal scarring, opacity, vascularisation and dystrophies or malignancy and infection of lacrimal glands. 2. Patients with systemic diseases such as diabetes mellitus, hepatic disorders, HIV and psychiatric disorders. 3. Pregnant and lactating women. 4.Patients on treatment with anticoagulants, antiglaucoma and anticholinergic drugs or drugs known to affect tear film. 5. Patients using topical corticosteroids (4 to 6 weeks prior to study enrolment). 6 . Patient allergic to fluorescein stain. A written informed consent was taken from the participants in their vernacular understood languages(Hindi or English), in the presence of a witness. A case study performa was filled up for each participant. Demographic information like name, age, sex, address, contact number of the patient and outpatient(O.P.D.) number, was collected. The Patient's symptoms were thoroughly evaluated and recorded. The symptoms relevant to dry eye were analysed using OSDI scores. The OSDI includes a scoring system to determine the severity of dry eye symptoms. A scale of 0 to 100 was used in assessment, with higher scores indicating greater severity of disease. The twelve questions of the OSDI were grouped into three sections. The sections assessed presence of symptoms over the last one week. Each question had a scale of 0 to 4 , indicating increasing severity. Each section's score was added to give a sum of scores for all questions answered. A score was then determined for each participant's OSDI using the following formula OSDI $=($ Sum of scores)/(Number of questions answered) $\mathrm{X} 25$ 
Detailed slit lamp examination(Slit lamp bio-microscopeHaag Streit BM 900) was done to exclude any lid conditions such as meibomitis, any eye lid deformities and lagophthalmos which may disturb the normal tear film. The Biomicroscopic Examination(BME) of the meibomian glands, lids, conjunctiva and tear film was performed at a slit lamp to grade the presence/severity of meibomian gland disease(MGD) and to assess signs of ocular surface abnormality and inflammation. The grading scale was categorised according to Foulks and Bron(Annexure III). For the purpose of statistical analysis, grades 2 or above were regarded as positive for the presence of ocular surface abnormality. The Tear film Break-Up Time(TBUT) assessment was done and the readings analysed. The tear film break-up time was defined as the interval between the last complete blink and the first appearance of a dry spot, or disruption in the tear film. A sterile fluorescein strip wetted with a drop of normal saline was instilled onto the bulbar conjunctiva as follows: with fixation directed inferonasaly, and the upper lid gently retracted the fluorescein strip was introduced at an approximate $30^{\circ}$ angle to the superior temporal bulbar conjunctiva and touched for 1-2 seconds, so that 1-2 mm of the flat side makes contact. The patient was instructed to blink naturally, without squeezing, several times to distribute the fluorescein. Within $10-30$ seconds of the fluorescein instillation, the patient was asked to stare straight ahead without blinking, until told otherwise. Slitlamp magnification was set at 10X. Cobalt blue light was used for observing the tear film over the cornea. Stopwatch was used to record time between last complete blink and first appearance of dry spot. Once TBUT was observed, patient was instructed to blink freely. Two readings were taken and averaged. The schirmer's I test was done and the reading noted. The test was done by placing the Schirmer's strip, made up of Whatman filter paper number 41 with dimensions $5 \mathrm{~mm}$ X $35 \mathrm{~mm}$. The initial $5 \mathrm{~mm}$ of the strip was folded and kept in junction of lateral one third and medial two third of the lower fornix of the eye. It was kept for 5 minutes. The wetting of the strip at the end of 5 minutes was noted using the scale present on the strip. The Conjunctival impression cytology(CIC) specimens were obtained from the inferior bulbar conjunctiva using a circular 0.22-micron pore size Millipore mixed cellulose acetate filter paper patch, $13 \mathrm{~mm}$ in diameter cut into two halves. The eye was anesthetized using one drop of $4 \%$ Xylocaine. The lacrimal lake at the inner canthus was dried with a cotton tip applicator. The filter paper was grasped with a blunt smooth edge forceps and applied to the inferior bulbar conjunctiva. The filter paper was pressed gently with an applanation head piece of Goldman's applanation tonometer held in the other hand. After 4 to 10 seconds, the paper strip was removed in a peeling fashion and the specimen was transferred to the glass slide coated with albumin paste by pressing the filter paper on it. The slide was labelled and numbered. The slide was put into Koplin jar containing fixative solution made up of Glacial acetic acid, Formaldehyde and Ethyl Alcohol in a 1:1:20 volume ratio. The slide was kept in fixative solution for 10 minutes and transported to the department of Pathlolgy. It was then stained with Periodic Acid-Schiff and counter stained with Hematoxylin and Eosin. The mounted slide was examined under the microscope with a 10x high-power field (HPF). After localization, the cells was then analysed with 40x HPF magnification. At least $10 \mathrm{HPF}$ were examined for goblet cells and epithelial cells. Grading and scoring were carried out using the criteria suggested by Nelson29. Grades 0 and 1 were regarded as normal, whereas grades 2 and 3 were considered to represent abnormal cytology. Study was approved by Institutional Ethical Committee.

\section{RESULTS}

A total of 142 subjects ( 74 cases and 68 controls) from 20 years of age to 60 years were included in the study. There were 51 males (35.9\%) and 91 females (64.1\%), with male to female ratio $1: 2$. There was a preponderance of females in our study in both the groups. However, the difference between males and females in different groups was not statistically significant $(p=0.840)$. Mean age of the participants was $38.61 \pm 12.67$ (minimum 20 years and maximum 60 years of age) in cases and $36.12 \pm 12.21$ (minimum 20 years and maximum 60 years of age) in controls. In the present study, $32.4 \%$ cases of dry eye were in age range of $20-30$ years, $27.5 \%$ in $31-40$ years, $22.5 \%$ in $41-50$ years and $17.6 \%$ in $51-60$ years. The DED was found to be more in females ( $64.9 \%$ of cases) than males (35.1\% of cases). The study included $22(29.7 \%)$ farmers, $15(20.3 \%)$ students/IT professionals, $10(13.5 \%)$ homemakers and 27(36.5\%) other professionals, in cases. Controls included 15(22.1\%) farmers, 15(22.1\%) students/IT professionals, 12 (17.6\%) home-makers and $26(38.2 \%)$ other professionals. Thus, it was observed that the dry eye was more common in patients indulging in outdoor activities. Conjuntival congestion was seen in $78.4 \%$ of cases and $58.8 \%$ of controls. Similarly, Normal conjunctiva was seen in $16.2 \%$ of cases and $38.2 \%$ of controls. The difference was significant $(p<0.05)$. For schirmer's test, $8.8 \%$ eyes of cases and $6.6 \%$ eyes of controls had schirmer values of $<5 \mathrm{~mm}$, similarly $12.8 \%$ of cases and $7.4 \%$ of controls had values between 5 and $10 \mathrm{~mm}$. The values of $>15 \mathrm{~mm}$ were observed in $70.3 \%$ of cases and $77.9 \%$ of controls. The difference was found not to be significant $(\mathrm{p}=0.379$ ). For control group, the mean was $24.32 \pm 10.088$, for case group, the mean was 23.34 \pm 11.662 . 
For TBUT test, $18.9 \%$ eyes of cases and $13.2 \%$ eyes of controls had TBUT value of $<5 \mathrm{sec}$, similarly $36.5 \%$ of cases and $24.3 \%$ of controls had values between 5 and 10 sec. The values of $>10 \mathrm{sec}$ were observed in $44.6 \%$ of cases and $62.5 \%$ of controls. For TBUT test, if value of $10 \mathrm{sec}$ was taken as cut-off for diagnosing a case of DED, then $55.4 \%$ eyes of cases and $37.5 \%$ eyes of controls had TBUT value of $=<10 \mathrm{sec}$, similarly $44.6 \%$ of cases and $62.5 \%$ of controls had values $>10 \mathrm{sec}$. For control group, the mean was $12.33 \pm 5.795$ in seconds, for case group, the mean was $10.05 \pm 5.498$ in seconds. The difference was found to be statistically significant ( $p$ value $<0.05$ ). For CIC test, by taking grade $0-1$ as normal and grade $2-3$ as abnormal cytology, $54.7 \%$ of cases and $84.6 \%$ of controls had normal cytology. Similarly, $45.3 \%$ of cases and $15.4 \%$ of controls had abnormal cytology. For control group, the mean was $0.84 \pm 0.69$, for case group, the mean was $1.39 \pm 0.787$. The difference between cases and controls was statistically significant ( $p$ value $<0.0001$ ). For BME test, by taking grade $0-1$ as normal and grade $2-3$ as abnormal, $57.4 \%$ of cases and $83.1 \%$ of controls had normal BME grades. Similarly, $42.6 \%$ of cases and $16.9 \%$ of controls had abnormal BME grades. For control group, the mean was $0.89 \pm 0.767$, for case group, the mean was $1.39 \pm 0.869$. The difference between cases and controls was found to be statistically significant( $p$ value $<0.0001$ ). The difference between case and control groups regarding age was found not to be statistically significant. Similar result was also observed for Schirmer's test. But for TBUT, CIC and BME the difference was found to be statistically significant. On cross tabulation, it was observed that $61.4 \%$ of subjects with abnormal impression cytology (grade 3,4) had Schirmer values $>15 \mathrm{~mm}$, while $79.6 \%$ of subjects with normal impression cytology(grade $0,1)$ had Schirmer values $>15 \mathrm{~mm}$. Similarly, it was found that $17.1 \%$ of subjects with abnormal cytology had Schirmer scores $<5 \mathrm{~mm}$ in comparison to $3.6 \%$ of subjects with normal cytology. The difference in both the groups was found to be statistically significant $(p<0.0001)$. The various parameters calculated from cross tabulation of Schirmer's test and CIC (taking CIC as gold standard) were; sensitivity of Schirmer's test was $30.7 \%$, specificity was $87.8 \%$, positive predictive value is $52.9 \%$ and negative predictive value was $73.8 \%$. On cross tabulation it was observed that $25 \%$ of subjects with abnormal impression cytology (grade 3,4) had TBUT values $>10 \mathrm{sec}$, while $65.8 \%$ of subjects with normal impression cytology(grade $0,1)$ had TBUT values $>10 \mathrm{sec}$. Similarly, it was also found that $37.5 \%$ of subjects with abnormal cytology had TBUT scores $<5 \mathrm{sec}$ in comparison to $6.5 \%$ of subjects with normal cytology. The difference in both the groups was found to be statistically significant $(\mathrm{p}<0.0001)$. The various parameters calculated from cross tabulation of TBUT and CIC(taking CIC as gold standard) were; sensitivity of TBUT test was $75 \%$, specificity was $65.8 \%$, positive predictive value was $49.6 \%$ and negative predictive value was $85 \%$. On cross tabulation it was observed that $52.3 \%$ of subjects with abnormal impression cytology(grade 3,4) had normal BME grade score(grade 0,1 ), while $77.6 \%$ of subjects with normal impression cytology(grade 0,1) had normal BME grade score (grade 0,1 ). Similarly, it was also found that $47.7 \%$ of subjects with abnormal impression cytology had abnormal BME grade score (grade 2,3) in comparison to $22.4 \%$ of subjects with normal impression cytology. The difference in both the groups was found to be statistically significant $(\mathrm{p}<0.0001)$. The various parameters calculated from cross tabulation of BME and CIC (taking CIC as gold standard) were; sensitivity of BME was $47.7 \%$, specificity was $77.6 \%$, positive predictive value was $48.8 \%$ and negative predictive value was $76.8 \%$. There was a positive correlation between CIC grades, OSDI scores and BME Grades (correlation coefficient, $r>0$ ). Increasing values of OSDI and BME was associated with increasing severity of conjunctival impression cytology. There was a negative correlation between CIC grades, Schirmer values and TBUT scores $(r$ $<0)$. The decreasing level of TBUT and Schirmer were associated with increasing severity of conjunctival impression cytology By taking cut-off value of Schirmer's test as $10 \mathrm{~mm}$ and TBUT as 10seconds together, the sensitivity, specificity and PPV were calculated (taking CIC as reference standard) as $81.8 \%, 61.2 \%$ and $48.6 \%$ respectively. While sensitivity, specificity and PPV of TBUT test alone were $75 \%, 65.8 \%$ and $49.6 \%$ respectively. Therefore, it can be concluded that though if TBUT and Schirmer's test are considered together for diagnosing DED, sensitivity(true positive rate) increases but specificity(true negative rate) decreases. In other words, TBUT alone is better predictor of DED than TBUT and Schirmer together. Taking CIC as gold standard, the accuracy of tear function tests in predicting morphological and cytological changes in the conjunctiva was TBUT $>$ BME $>$ Schirmer. In decreasing order, the sensitivity of tear function tests in diagnosing dry eye was TBUT $>$ BME $>$ Schirmer, and the specificity was Schirmer $>\mathrm{BME}$ $>$ TBUT. In our study ROC curves were drawn taking CIC as reference standard and area under the curve was calculated(AUC). AUC was 0.775 for TBUT, 0.730 for OSDI, 0.693 for BME and 0.653 for Schirmer's test. Therefore, the diagnostic accuracy was $\operatorname{TBUT}(0.775)$ $>\mathrm{OSDI}(\mathrm{AUC}=0.730)$

$>$ BME(AUC $=0.693)$

$>$ Schirmer $(\mathrm{AUC}=0.853)$. 


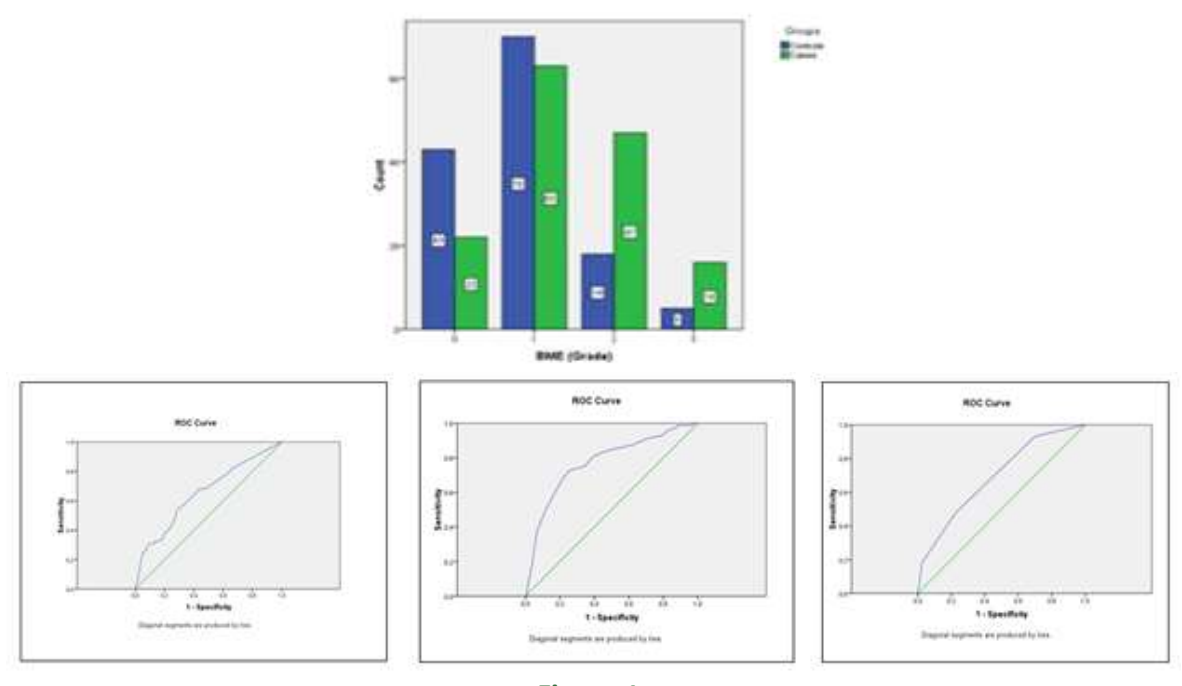

Figure 1:

\section{DISCUSSION}

The dry eye was found to be more in females $(64.9 \%$ of cases) than males( $35.1 \%$ of cases $)$. Similarily There was a female preponderance in dry eye disease in a study by Shreshta E et al. ${ }^{17}$ A higher proportion of females had aqueous tear deficiency than men and an increased prevalence of all categories of treated dry eye occurs in women, compared with men. ${ }^{18}$ The higher prevalence of dry eye disease in females was found in other studies. Moss SE et al. ${ }^{19}$ reported dry eye more in women(16.7\%) than men $(11.4 \%)$ and similarly Gupta $\mathrm{N}$ et al. ${ }^{20}$ reported dry eye more in women $(27 \%)$ than men( $12 \%)$. In a study by Basak SK et al. ${ }^{21}$ dry eye disease was present in $51.9 \%$ women and $48.1 \%$ men. But Schein et al. ${ }^{22}$ reported no correlation between dry eye and age or sex. In India, Sinha et al. ${ }^{23}$ and Bhatnagar et al. ${ }^{24}$ reported an incidence of dry eye of $46 \%$ and $10.58 \%$ respectively. Sahai et al. ${ }^{25}$ studied the prevalence of dry eye in a hospital-based population in India. It was found to be $18.4 \%$ based upon objective measurements. It was significantly higher in females(22.8\%) than males(14.9\%). In our study $31.1 \%$ of cases of dry eye were in the age group of 20-30 years, while $27 \%$ were in the age group of $41-50$ years. Only $16.2 \%$ were in the age group of 51-60 years. Shrestha E et al. ${ }^{17}$ in their study also observed maximum number of cases within the range of 21-30 years. The higher number of cases of dry eye in younger age group, in our study could be due to excessive use of computers and smartphones. For schirmer's test, $8.8 \%$ eyes of cases and $6.6 \%$ eyes of controls had schirmer values of $<5 \mathrm{~mm}$, similarly $12.8 \%$ of cases and $7.4 \%$ of controls had values between 5 and $10 \mathrm{~mm}$. The values of $>15 \mathrm{~mm}$ were observed in $70.3 \%$ of cases and $77.9 \%$ of controls. The difference was found not to be significant $(\mathrm{p}=0.379)$. For control group, the mean was $24.32 \pm 10.088$, for case group, the mean was 23.34 \pm 11.66 . similar findings were observed in Danjo et al. ${ }^{26}$
Rahul Bhargava and Prachi Kumar ${ }^{27}$ found the sensitivity and specificity of Schirmer's test as $38.2 \%$ and $82 \%$ respectively. In our study, sensitivity, specificity, PPV and NPV of TBUT test were 75\%, 65.8\%, 49.6\% and $85 \%$ respectively. Our results were similar to that of study by Kumar et al..88sand Rahul Bhargava and Prachi Kumar. ${ }^{27}$ In a study by Danjo et $a l .{ }^{26}$ the diagnostic usefulness of TBUT was found to be superior to that of Schirmer's I test, which was similar to our study. In our study, out of 74 cases, $45.34 \%$ had abnormal impression cytology; amongst these $(39.2 \%$ had Nelson grade 2 and $6.1 \%$ Nelson grade 3 changes, respectively); whereas amongst 68 controls, only $15.4 \% \%$ (Nelson grade 2 and 3 ) had abnormal cytology and $84.6 \%$ had normal cytology(Nelson grade 0 and 1). These results were similar to those obtained by Shrestha E et al., ${ }^{17}$ Kumar P et al. ${ }^{28}$ and Bhargava et al. ${ }^{27}$ In our study ROC curves were drawn taking $\mathrm{CIC}$ as reference standard and area under the curve was calculated (AUC). AUC was 0.775 for TBUT, 0.730 for OSDI, 0.693 for BME and 0.653 for Schirmer's test. Therefore, the diagnostic accuracy was $\operatorname{TBUT}(0.775)$ $>\mathrm{OSDI}(\mathrm{AUC}=0.730)$

$>\mathrm{BME}(\mathrm{AUC}=0.693)$

$>$ Schirmer $(\mathrm{AUC}=0.853)$. The results of our study were similar to what was seen by Kumar P et al. ${ }^{28}$ In a study by Jonathan E Moore et al. ${ }^{29}$ Sood et al. ${ }^{30}$ demonstrated a strong correlation between assessments made by tear func $\neg$ tion tests like Schirmer's and CIC results. In a study by Kumar $\mathrm{P}$ et al. ${ }^{28}$, In decreasing order, the sensitivity of tear function tests in diagnosing dry eye was TBUT $>$ Schirmer's test $>$ RBS, and the specificity was Schirmer's test $>$ TBUT $>$ RBS. CIC was considered to be the gold standard. Routine tear function tests like Schirmer, TBUT and RBS are commonly used by eye care professionals worldwide for evaluation of dry eye syndrome; these tests can be performed in all settings, are inexpensive and less time consuming than CIC. However, some of these tests 
are poorly standardized, lack well defined cut-off values, do not correlate well with dry eye symptoms, are inaccurate and unrepeatable. CIC can be used in conjunction with techniques like light microscopy, flow cytometry, RT-PCR amplification and immunohistochemistry, to aid in diagnosis and providing insight into mechanism and pathogenesis of DED. ${ }^{31}$

\section{CONCLUSION}

TBUT and BME hold good diagnostic accuracy for dry eye evaluation in patients.

\section{REFERENCES}

1. Bron AJ, Introduction.SurvOphthalmol. 2001;45:197.

2. The epidemiology of dry eye disease: report of the Epidemiology Subcommittee of the International Dry Eye WorkShop.Ocul Surf 2007;5:93-107.

3. Research in dry eye: report of the Research Subcommittee of the International Dry Eye WorkShop .Ocul Surf 2007;5:179-93.

4. The definition and classification of dry eye disease: Report of the definition and classification subcommittee of the International Dry Eye Workshop (2007). Ocul Surf. 2007; 5:7592.

5. Bron AJ. Reflections on the tears. Eye 1997; 11:583-602.

6. Kallarackal GU, Ansari EA, Amos N, Martin JC, Lane C, Camilleri JP. A comparative study to assess the clinical use of Fluorescein Meniscus Time (FMT) with tear break up time (TBUT) and Schirmer's tests (ST) in the diagnosis of dry eyes. Eye 2002;16:594-600.

7. Dogru M, Tsubota K. New insights into the diagnosis and treatment of dry eye. Ocul Surf. 2004;2:59-75.

8. Begley CG, Caffery B, Chalmers RL, Mitchell GL. Dry Eye Investigation (DREI) Study Group, Use of the dry eye questionnaire to measure symptoms of ocular irritation in patients with aqueous tear deficient dry eye. Cornea 2002;21:664-70.

9. Dart J. Impression cytology of the ocular surface: research tool or routine clinical investigation?Br J Ophthalmol.1997;81:930.

10. Nelson JD, Wright JC. Conjunctival goblet cell densities in ocular surface disease. Arch Ophthalmol 1984;102:1049-51.

11. Oroza MA, Pérez-Esteban A, Murube J. Topographical distribution of ocular surface cells by the use of impression cytology. ActaOphthalmol. 1991;69:371-76.

12. Barros JN, Almeida SA, Lowen MS, Cunha MC, Gomes JP.Impression cytology in the evaluation of ocular surface tumors: review article.Arq Bras Oftalmol. 2015;78(2):126-32

13. Tseng SG. Staging of conjunctival squamous metaplasia by impressioncytology. Ophthalmology .1985;92:728-33.

14. Natadisastra G, Wittpenn JR, West KP , Alfred S. Impression cytology for detection of vitamin A deficiency. Arch Oph $\neg$ thalmol1987;105:1224-28.
15. Puangsricharern V, Tseng SC. Cytologic evidence of corne $\neg$ al diseases with limbal stem cell deficiency. Ophthalmolo $\neg$ gy 1995;102:1476-85.

16. Lee GA, Hirst LW. Ocular surface squamous neoplasia. SurvOphthalmol1995;39:429-50.

17. Shrestha ,Shrestha J K, Shayami G,Chaudhary M. Conjunctival impression cytology in dry eye syndrome. Nepal J Ophthalmol 2011; 3 ( 5 ): 39-44

18. Yazdani C, McLaughlin T, Smeeding JE, Walt J. Prevalence of treated dry eye disease in a managed care population. ClinTher 2001;23:1672-82.

19. Moss SE, Klein R, Klein BE. Prevalence of and risk factors for dry eye syndrome. Arch Ophthalmol. 2000;118:1264-8.

20. Gupta N, Prasad I, Jain R, D'Souza P. Estimating the prevalence of dry eye among Indian patients attending a tertiary ophthalmology clinic. Ann Trop Med Parasitol. 2010 Apr;104(3):247-55.

21. Basak SK, Pal PP, Basak S, Bandyopadhyay A, Choudhury S, Sar S. Prevalence of dry eye diseases in hospital-based population in West Bengal, Eastern India. J Indian Med Assoc.2012;110:789-94.

22. Schein OD, Tielsch JM, Munoz B, Bandeen-Roche K, West S.. Relation between signs and symptoms of dry eye in the elderly. Ophthalmology .1997;104:1395-401.

23. Sinha R, Agarwal P, Kumar C. Small incision cataract surgery: Review of journal abstracts. Indian J Ophthalmol 2009;57:7982.

24. Bhatnagar KR, Sapovadia A, Gupta D, Kumar P, Jasani H. Dry eye syndrome: A rising occupational hazard in tropical countries. Med J DY Patil Univ. 2014;7:13-8.

25. Sahai A, Malik P. Dry Eye: Prevalence and Attributable Risk Factors in a Hospital-Based Population. Indian J Ophthalmol 2005;53:87-91.

26. Danjo Y. Diagnostic usefulness and cut off value of Schirmer's I test in the Japanese diagnostic criteria of dry eye. Graefes Arch ClinExpOphthalmol 1997;235:761-6.

27. Bhargava R, Kumar P .Can Conjunctival Impression Cytology be the First Line Diagnostic Test for Evaluation of Dry Eye Syndrome? Enliven: ClinOphthalmol Res 2015;1(1): 004.

28. Kumar P, Bhargava R , Kumar M, Ranjan S, Kumar M, VermaP.The correlation of routine tear function tests and conjunctival impression cytology in dry eye syndrome Korean $\mathrm{J}$ Ophthalmol 2014;28:122-29.

29. Moore J E, Graham J E, Goodall E A, Dartt E A, Leccisotti A, Mc Gilligan VE et al... Concorddance between common dry eye diagnostic tests Br J Ophthalmol. 2009;93(1):66-72.

30. Sood S, Shukla R, Nada M, Khurana, A.K., Arora, B. Comparison of tear film profile, conjunctival impression cytology, and conjunctival biopsy in patients with dry eye. Asian J Ophthalmol.2006;8:24-27.

31. Brignole-Baudouin F, Ott AC, Warnet JM, Baudouin C. Flow cytometry in conjunctival impression cytology: a new tool for exploring ocular surface pathologies 1996. Exp Eye Res. 1996; 78:473-81.

\section{Source of Support: None Declared Conflict of Interest: None Declared}

Policy for Articles with Open Access:

Authors who publish with MedPulse International Journal of Ophthalmology (Print ISSN: 2250-7575) (Online ISSN: 2636-4700) agree to the following terms: Authors retain copyright and grant the journal right of first publication with the work simultaneously licensed under a Creative Commons Attribution License that allows others to share the work with an acknowledgement of the work's authorship and initial publication in this journal.

Authors are permitted and encouraged to post links to their work online (e.g., in institutional repositories or on their website) prior to and during the submission process, as it can lead to productive exchanges, as well as earlier and greater citation of published work. 